\title{
Comparative effectiveness of photobiomodulation and manual therapy alone or combined in TMD patients: a randomized clinical trial
}

\author{
Fernanda Thomé BROCHADO(a) \\ Luciano Henrique de JESUS(a) \\ Vinicius Coelho CARRARD(a) \\ Angelo Luiz FREDDO(b) \\ Karen Dantur CHAVES(c) \\ Manoela Domingues MARTINS(a)
}

aniversidade Federal do Rio Grande do Sul - UFRGS, School of Dentistry, Department of Oral Pathology, Porto Alegre, RS, Brazil.

bUniversidade Federal do Rio Grande do Sul - UFRGS, School of Dentistry, Department of Buco Maxillofacial Surgery and Traumatology, Porto Alegre, RS, Brazil.

'Universidade Federal do Rio Grande do Sul - UFRGS, School of Dentistry, Department of Conservative Dentistry, Porto Alegre, RS, Brazil.

Declaration of Interest: The authors certify that they have no commercial or associative interest that represents a conflict of interest in connection with the manuscript.

Corresponding Author: Manoela Domingues Martins manomartins@gmail.com

htrps://doi.org/10.1590/1807-3107bor-2018.vol32.0050

Submitted: September 08, 2017

Accepted for publication: April 10, 2018

Last revision: April 25, 2018
Abstract: The effectiveness of photobiomodulation (PBM) and manual therapy (MT), alone or combined (CT), were evaluated in pain intensity, mandibular movements, psychosocial aspects, and anxiety symptoms of temporomandibular disorder (TMD) patients. Fifty-one TMD patients were randomly assigned to three groups: the PBM group $(n=18)$, which received PBM with $808 \mathrm{~nm}, 100 \mathrm{~mW}, 13.3 \mathrm{~J} / \mathrm{cm}^{2}$, and $4 \mathrm{~J}$ per point; the MT group $(n=16)$ for 21 minutes each session on masticatory muscles and temporomandibular joint TMJ; and the CT group $(\mathrm{n}=17)$, applied during twelve sessions. Seven evaluations were performed in different moments using visual analogue scale (VAS), Research Diagnosis Criteria for Temporomandibular Disorders (RDC/TMD) Axis I and II, and Beck anxiety inventory (BAI). All groups demonstrated reductions in pain and improvement in jaw movements during treatment and at follow-up (< 0.001). The assessment of psychosocial aspects of TMD, comparing baseline and follow-up in all treatment groups, revealed that treatment did not promote modification in the intensity of chronic pain ( $p>0.05)$. However, depression symptoms showed a reduction in PBM and CT groups $(\mathrm{p} \leq 0.05)$. All treatments promoted reduction in physical symptoms with and without pain and enhancement of jaw disabilities ( $\mathrm{p} \leq 0.05)$. MT promotes improvement in 5 functions, PBM in 2 , and CT in 1 ( $\mathrm{p}<0.001)$. BAI analysis revealed that all treatments lead to a reduction in anxiety symptoms $(p \leq 0.05)$. All protocols tested were able to promote pain relief, improve mandibular function, and reduce the negative psychosocial aspects and levels of anxiety in TMD patients. However, the combination of PBM and MT did not promote an increase in the effectiveness of both therapies alone.

Keywords: Temporomandibular Joint Diseases; Low-level Laser Therapy; Treatment, Manual Therapies.

\section{Introduction}

Temporomandibular disorders (TMDs), considered the major cause of non-dental facial pain, involve a wide range of symptoms in masticatory muscles and temporomandibular joints. ${ }^{1,2}$ Diagnosis is usually delayed due to the multifactorial etiology, and the lack of assessment devices and parameters 
for studies. ${ }^{2}$ Most studies that evaluate TMD treatment modalities use only Visual Analogue Scale (VAS) and mouth opening analysis, neglecting important aspects such as chronic pain, depression, anxiety, and other symptoms. To increase the consistency between studies, the Research Diagnosis Criteria for Temporomandibular Disorders (RDC/TMD) was developed and has been used worldwide as a gold standard for TMD evaluation. ${ }^{2}$ The measure is a dual-axis system, where Axis I involves the clinical examination resulting in TMD classification into three groups: 1) muscle disorders, including myofascial pain, with and without mouth opening limitation; 2) disk displacement, with or without mouth opening reduction or limitation; and 3) arthralgia, arthritis, and/or arthrosis. Axis II assesses functional jaw disability, psychological status, and psychosocial functioning. ${ }^{3,4}$ Axis II is very important, since around $75 \%$ of patients suffer from chronic symptoms, with negative biopsychosocial consequences. ${ }^{4}$

Therapies such as photobiomodulation therapy (PBM) and manual therapy (MT) aim to relieve TMD symptoms and improve function, but there is no consensus about their efficacy. ${ }^{5,6,7,8,9,10,11,12,13,14,15,16,17,18,19,20,21,22,23,24,25,26}$ PBM treatment involves the use of laser or lightemitting diodes (LED), with wavelengths ranging from the visible light spectrum $(\lambda=400 \mathrm{~nm})$ to the infrared rays $(\lambda=1,064 \mathrm{~nm})$ and with power below $500 \mathrm{~mW} .^{8,11}$ It has been used to reduce pain, inflammation, edema, and to regenerate damaged tissues such as bones and tendons. ${ }^{11}$ PBM involves the absorption of photons by the natural chromophore cytochrome c oxidase that catalyzes the metabolic oxygen reduction reaction, accelerating respiratory metabolism and resulting in cellular proliferation, antioxidant and redox-regulation, prevention of cell death, reestablishment of cellular metabolism, and reduction of pain and inflammation. ${ }^{11}$ Previous studies have shown the beneficial effects of PBM in TMD management such as reduction in muscle and joint pain and improvement in jaw movements and chewing. ${ }^{1,717,20,22,24}$ However, some studies indicate that the efficacy of laser in the treatment of TMD is controversial, probably due to a placebo effect, and differences in the laser irradiation parameters or the criteria used for the classification and evaluation of TMD. $8,15,16,20,23$

MT is another commonly used treatment based on joint manipulation and muscle mobilization techniques, which promote the release of inhibitory neurotransmitters, opioids, and non-opioid substances, improving movements, muscle relaxation, and providing pain relief. ${ }^{10,26}$ Some studies performed MT in TMD patients and presented conflicting results. $10,13,25,26$

The association of therapies is suggested to show better results, but few studies have been performed using the combination of PBM with other therapies like splint therapy ${ }^{12}$ and/or transcutaneous electrical neural stimulation. ${ }^{13}$ Based on the above aspects, it is unclear whether PBM and MT, alone or combined, are effective to treat TMD. We hypothesized that all therapies could promote pain relief and improve masticatory function, especially when using the combined protocol. Thus, the aim of the present study was to compare the effectiveness of PBM and $\mathrm{MT}$, alone or combined, in the treatment of pain, movement restriction, psychosocial disorders, and anxiety symptoms of TMD.

\section{Methodology}

\section{Study design}

The present single-center, randomized, singleblind study received approval from the Human Research Ethics Committee (CEP/UFRGS 1.541.360 and CAEE protocol 52651416.1.0000.5347). All participants signed a statement of informed consent prior to any clinical procedure.

\section{Sample size calculation}

Sample size calculation was based on analysis of pain variability (Visual Analogue Scale-VAS), as published by Carrasco et al., ${ }^{9}$ with a VAS standard deviation (SD) of $1.64 \mathrm{~cm}$ (experimental group) and $2.28 \mathrm{~cm}$ (control group). A difference of $2.5 \mathrm{~cm}$, $80 \%$ power, and $5 \%$ level of significance were used. A minimum of 10 subjects in each experimental group was determined. Considering a follow up of 90 days and an estimated $60 \%$ adherence, some additional individuals were recruited in each group. 
The software Winpepi version 10.5 was used for this calculation.

\section{Participants, randomization, and blinding}

Fifty-four adult patients with TMD were enrolled in the study between May 2016 and November 2016. For inclusion in this study, patients had to be 21 years or older, be diagnosed with myogenic and arthrogenic TMD based on RDC/TMD Axis I analysis, present pain in temporomandibular joint (TMJ) and limited mouth opening. The exclusion criteria were current dental therapies that could affect TMJ, rheumatic diseases, and use of antiinflammatory drugs and muscle relaxants. From all patients, 3 were not included in the study due to rheumatic diseases $(n=1)$ and dental and medical treatments $(n=2)$. Therefore, 51 patients were randomly assigned to photobiomodulation group (GPBM) ( $\mathrm{n}=18)$, manual therapy group (GMT) $(n=16)$, or combined therapy group (GCT) $(n=17)$. Randomization was performed by the same professional who applied the therapies, using a card system that maintains complete randomness of the assignment of a subject to a particular group. This randomization approach is simple and easy to implement in a clinical research. Only one researcher knew in which group the patients were allocated and performed all the treatments (FTB). A blinded researcher performed all the evaluations (LHJ). The patient was aware of the treatment. Figure 1 displays the study flowchart.

\section{Clinical procedures and evaluations}

At baseline (D0), all patients were evaluated for symptoms score using VAS, RDC/TMD (Axis I and II), Beck Anxiety Inventory (BAI).

After randomization, all patients were submitted to treatment and were reevaluated once a week during the treatment period (D7, D14, D21, D28). This evaluation involved the recording of VAS and RDC/TMD (Axis I) analysis. The follow-up period was considered 4 weeks (D60) and 8 weeks (D90) after the treatment. At $\mathrm{D} 90$ patients were analyzed regarding VAS, RDC/TMD (Axis I and II), BAI.
Evaluations were performed by a single calibrated professional who was blinded to the allocation of the participants to the different treatment groups. All individuals were warned to not take any analgesics or anti-inflammatory medication during the study and inform the professional if they started any medical treatment.

\section{Symptom analysis}

Symptom scores were determined using a VAS, in all evaluation periods of the study. VAS score is a unidimensional measure used in epidemiologic and clinical research to evaluate the intensity or frequency of various symptoms especially pain. Consist in a straight horizontal line of fixed length, usually $100 \mathrm{~mm}$, orientated from the left (no pain) to right (most pain). Pain was considered the primary study variable.

\section{Axis I: TMD subgroup and assessment of jaw movements}

Physical evaluation was performed following Axis I of RDC/TMD, which provides information on jaw movement, pain, muscle palpation, and joint noises. Axis I divides TMD into 3 groups as follows: (GI) muscle disorders including myofascial pain with and without mouth opening limitation; (GII) disk displacement with or without mouth opening limitation; (GIII) arthralgia, arthritis, and arthrosis. The exam was performed as previously described by Rodrigues et al., in all evaluation periods.

\section{Axis II: Assessment of psychosocial aspects}

Axis II of RDC/TMD provides information on the psychosocial aspects of TMD and classifies patients into 4 domains: intensity of chronic pain and disability (D1), degree of depression symptoms (D2), nonspecific physical symptoms scale (D3), and limited mandibular function (D4). The nonspecific physical symptoms scale was subdivided into nonspecific physical symptoms with pain (D3a) and nonspecific physical symptoms without pain (D3b). As previously described by Rodrigues et al., 7 Axis II was applied at baseline and follow-up. None of the patients was previously evaluated by a specialist for depression diagnosis. 


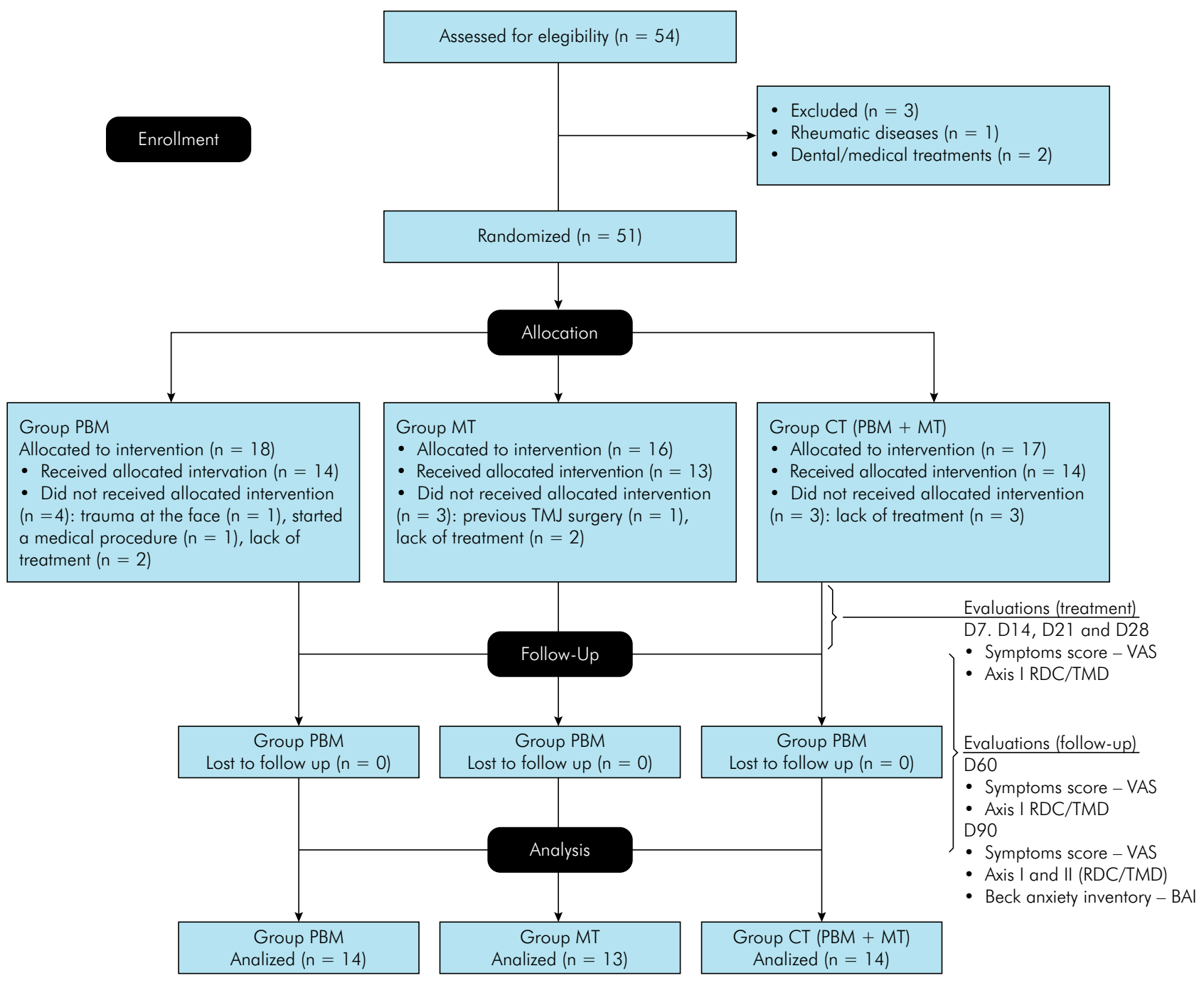

Figure 1. Flowchart showing subject enrollment and follow-up.

\section{Beck anxiety inventory-BAl}

Anxiety analyses were done using BAI, applied at baseline and follow-up. BAI is a 21 multiple-choice questionnaire regarding common symptoms of anxiety. The score ranges from 0 to 63 and is classified as minimal anxiety (score: 0-10), mild anxiety (score: 11-20), moderate anxiety (score: 21-30), and severe anxiety (score: 31-63).

\section{Photobiomodulation group (GPBM)}

PBM was applied by a single professional using a continuous wave of GaAlAs diode laser (MMOptics Recover, São Paulo, Brazil), with a wavelength of 808 $\mathrm{nm}$. Irradiation was performed in punctual contact mode with a spot size of $0.03 \mathrm{~cm}^{2}$, power output of $100 \mathrm{~mW}$, irradiance of $3.33 \mathrm{~W} / \mathrm{cm}^{2}$, radiant exposure of $133 \mathrm{~J} / \mathrm{cm}^{2}, 40$-s exposure time per point, and 4 $\mathrm{J}$ of total energy per point. PBM was applied 12 times ( 3 times a week for 4 consecutive weeks). The output power of the equipment was checked using a power meter (Laser Check; MMOptics LTDA, São Paulo, Brazil). During therapy, patients were in the supine position and received PBM in 12 points: 5 in the TMJ region (superior, anterior, lateral, posterior, and postero-inferior to the condyle) and 7 in muscles [temporal (anterior, middle, and posterior), masseter (upper, middle, and lower portion), and insertion of the medial pterygoid], according to the protocol used by Ahari et al. ${ }^{1}$ and Rodrigues et al. ${ }^{7}$ (Figure 2). The protocol results in $48 \mathrm{~J}$ per point or $576 \mathrm{~J}$ per each side of the face after 4 consecutive weeks. 


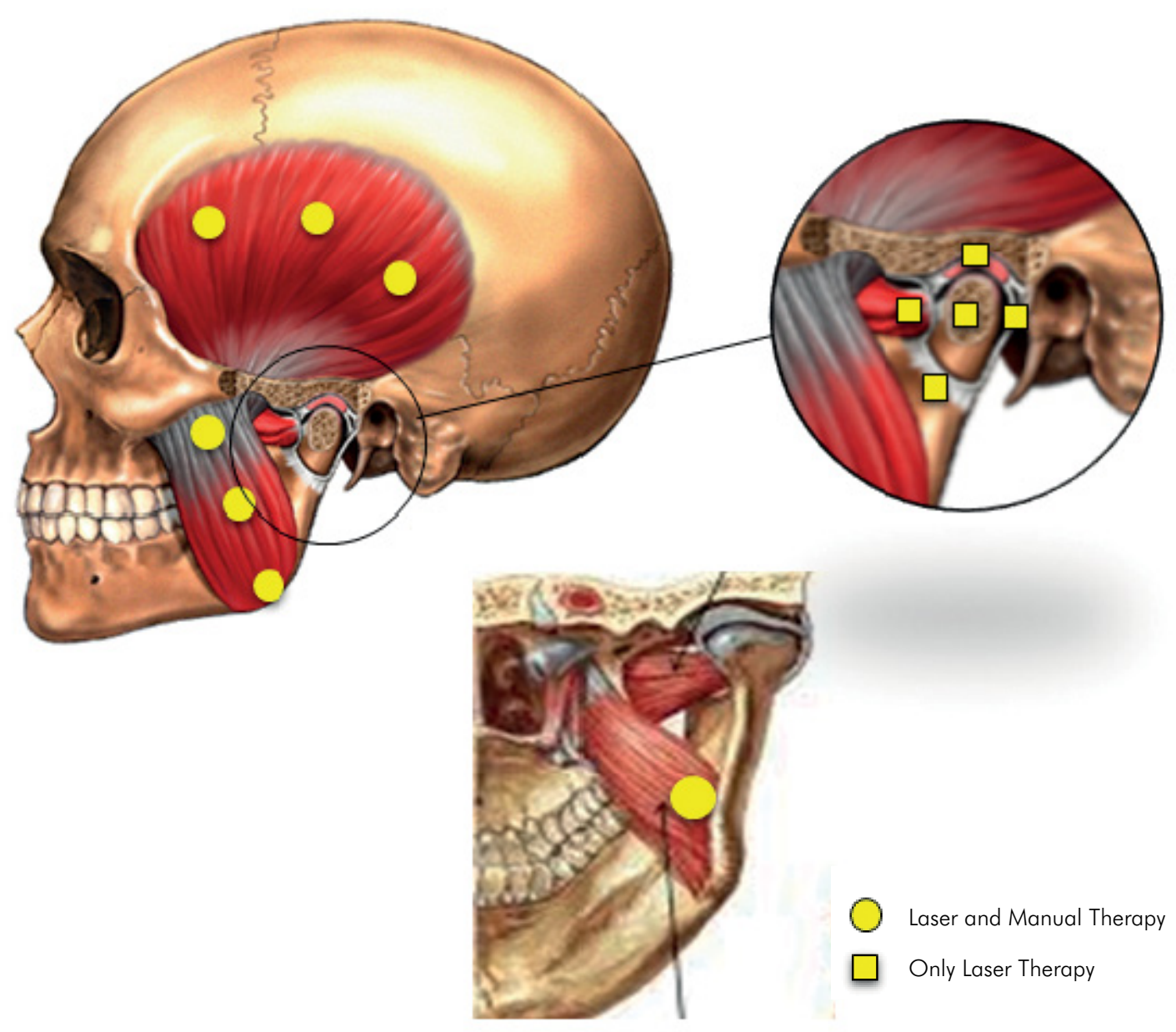

Figure 2. Schematic representation of the points of laser and manual therapy application.

\section{Manual Therapy Group (GMT)}

Patients were submitted to 3 weekly 21-minute sessions of MT on masticatory muscles and TMJ for 4 consecutive weeks. All procedures were performed by a physiotherapist who had undergone a training exercise for the administration of the protocol adapted from Biasotto-Gonzalez et al. ${ }^{14}$

For extraoral MT, patients were in the supine position while the professional performed circular movements with his fingers, sliding and compressing, from proximal to distal areas of the face, with constant and progressive pressure compatible with the situation of each tissue and the sensitivity of each individual. Temporal, masseter, and medial pterygoid muscles from both sides were submitted to MT for 3 minutes in each muscle group. Then, from the opposite side of the treatment site, the professional performed circular movements in the intraoral region of the masseter and lateral wall for 3 minutes each side. ${ }^{14}$

MT was performed on the TMJ with the professional positioned on the opposite side of the mobilization, by placing the thumb on the second or third molar (if present) and performing a caudal distraction with anterior projection, intermittently for 1 minute and 3 repetitions. MT was administered 3 times a week for 4 consecutive weeks ${ }^{14}$ (Figure 2).

\section{Combined therapy group (GCT)}

In each session, patients were submitted to PBM and MT protocols 3 times a week for 4 consecutive weeks.

\section{Statistical analysis}

Statistical analysis was carried out using SPSS version 21.0 (SPSS Inc., Chicago, Illinois). Data were 
analyzed using a generalized estimating equations (GEE) test, which combined tests for treatment differences and changes in treatment response over time. The GEE followed by Bonferroni's post hoc test was used to determine the significance of differences between therapies overtime. All statistical tests were performed with a significance level of $5 \%(p \leq 0.05)$. The statistical tests used considered the correlated nature of the repeated measures of each patient. Analyses were based on intention-to-treat.

\section{Results}

Table 1 summarizes the demographic characteristics of the 41 TMD patients evaluated during the study. TMD was more prevalent in females $(95.1 \%)$ with a mean age of 44 years $( \pm 17.1)$, ranging from 21 to 77 years old.

\section{Assessment of pain}

The VAS data obtained in all periods of evaluation (baseline, treatment, and follow-up) are demonstrated in Figure 3 (A and B). All patients had symptoms at baseline with an overall mean VAS score of 4.6 $( \pm 0.5)$, with no statistically significant difference between groups. All groups experienced a significant reduction in pain by D14 ( $\mathrm{p}<0.001)$. The change in mean VAS scores did not differ significantly between groups during evaluation time. In D90, all groups maintained a stable mean similar to D28 ( $<<0.05)$.

\section{Axis I: TMD subgroup}

The classification of TMD, based on Axis I, revealed that all patients were diagnosed as GI (muscle disorders) and GIII (arthralgia, arthritis, and arthrosis), exhibiting a combination of myogenic

\section{Axis II: Psychosocial aspects}

The assessment of psychosocial aspects comparing D0 and D90 in all treatment groups are demonstrated on Tables 4, 5, 6, and 7. D1 analysis revealed no significant difference in all groups between D0 and D90 (Table 4). D2 evaluation showed reduction in GPBM and GCT (Table 5). D3a and D3b analyses revealed reductions in physical symptoms with all treatments (Table 6 and 7).

The analysis of $\mathrm{D} 4$ denotes that the functions most affected in our patients were chewing, eating hard foods, smiling/laughing, cleaning teeth or face, and yawning. At D90 was observed an improvement of chewing (GMT), exercising (GCT), eating hard foods (GPBM and GMT), eating soft foods (GMT), smiling/ laughing (GPBM and GMT), and swallowing (GMT). MT promotes improvement in 5 disabilities, PBM in 2 , and CT in 1 (Table 8).

\section{Beck anxiety inventory}

The overall mean BAI score at D0 was 17.86 in GPBM, 14.92 in GMT (both mild anxiety), and 31.21 (severe anxiety) in GCT. At D90, all groups showed reduction in anxiety (Table 9).

\section{Discussion}

TMD has been considered a complex disease of multifactorial etiology and multifaceted treatment. Unfortunately, the delay in the correct diagnosis often leads TMD patients to chronic pain, characterized by an exacerbation of symptoms. ${ }^{10}$ Several studies attempted to identify the best methods for diagnosis and treatment of this condition, although no gold standard of treatment was found. ${ }^{5,6,12}$ Different

Table 1. General characteristics of patients at baseline.

\begin{tabular}{lcccc}
\hline Baseline characteristics & PBM & MT & CT & TOTAL \\
\cline { 2 - 5 } & $(\mathrm{n}=14)$ & $(\mathrm{n}=13)$ & $\mathrm{n}=14)$ & $(\mathrm{n}=41)$ \\
\hline Gender $-\mathrm{n}(\%)$ & & & & \\
Male & $0(0)$ & $1(2.4)$ & $1(2.4)$ & $13(2.9)$ \\
$\quad$ Female & $14(36.6)$ & $12(29.3)$ & $13(29.3)$ & $39(95.1)$ \\
Age - year (mean SD) & $45.7( \pm 15.7)$ & $41.2( \pm 20.3)$ & $46.5( \pm 14.4)$ & $44.5( \pm 17.1)$ \\
Duration of pain & $8.8( \pm 6.9)$ & $10.1( \pm 12.8)$ & $9.2( \pm 9)$ & $9.4( \pm 9.8)$ \\
\hline
\end{tabular}


3A

\begin{tabular}{|c|c|c|c|c|c|c|c|c|}
\hline \multirow{2}{*}{$\begin{array}{l}\text { Mean } \\
\text { (IC95\%) }\end{array}$} & \multicolumn{2}{|c|}{ Baseline } & \multicolumn{3}{|c|}{ Treatment } & \multicolumn{2}{|c|}{ Follow up } & \multirow{2}{*}{$p$-value } \\
\hline & D0 & D7 & D14 & D21 & D28 & D60 & D90 & \\
\hline PBM & $4.1^{a}[2.91-5.38]$ & $3.3^{a}[1.89-4.68]$ & $1.9^{\mathrm{b}}[1.10-2.76]$ & $1.8^{\mathrm{b}}[0.77-2.80]$ & $1.1^{\mathrm{b}}[0.43-1.71]$ & $0.8^{b}[0.19-1.39]$ & $1.6^{b}[0.96-2.27]$ & $<0.001$ \\
\hline MT & $4.4^{a}[2.46-6.31]$ & $2.8^{a}[1.51-4.03]$ & $1.7^{\mathrm{b}}[0.71-2.68]$ & $0.5^{b}[0.03-1.04]$ & $1.3^{\mathrm{b}}[0.17-2.44]$ & $0.6^{\mathrm{b}}[0116-1.07]$ & $0.9^{\mathrm{b}}[0.17-1.66]$ & $<0.001$ \\
\hline CT & $5.2^{a}[3.75-6.61]$ & $3.4^{\circ}[2.23-4.49]$ & $2.4^{\mathrm{b}}[1.72-3.13]$ & $1.9^{b}[1.12-2.59]$ & $0.9^{b}[0.43-1.43]$ & $1.2^{b}[0.72-1.71]$ & $1.9^{\mathrm{b}}[0.79-2.92]$ & $<0.001$ \\
\hline$p$-value & 0.30 & 0.80 & 0.49 & 0.06 & 0.81 & 0.30 & 0.39 & \\
\hline
\end{tabular}

GEE Model: normal distribution with identify link and ndependent correlation matrix; ${ }^{*}$ Different lowercase letters on lines (intra group analysis) denote significant difference $(p<0.05)$.

3B

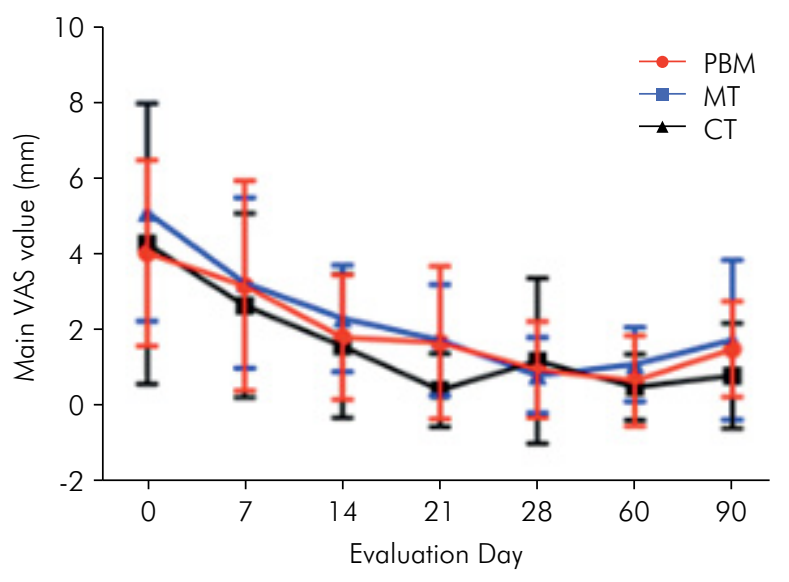

Figure 3 (A e B). Mean symptom and 95\%Cl of VAS scores in PBM, MT, and CT groups during evaluation and follow-up period.

Table 2. RDC/TMD Axis I. Assessment of clinical TMD conditions before and after treatments.

\begin{tabular}{lcccccc}
\hline \multirow{2}{*}{ Axis I - n (\%) } & \multicolumn{3}{c}{ PBM } & \multicolumn{3}{c}{ CT } \\
\cline { 2 - 7 } GI & Baseline (DO) & Follow up (D90) & Baseline (DO) & Follow up (D90) & Baseline (DO) & Follow up (D90) \\
\hline GII & $14(34.2)$ & $9(22)$ & $13(31.7)$ & $7(17.1)$ & $14(34.1)$ & $13(31.7)$ \\
GIII & $2(4.8)$ & $1(2.4)$ & $1(2.4)$ & $0(0)$ & $1(2.4)$ & $0(0)$ \\
p-value & $14(34.2)$ & $8(19.5)$ & $13(31.7)$ & $6(14.6)$ & $14(34.1)$ & $7(17.1)$ \\
\hline
\end{tabular}

GI: muscle disorders; Gll: displacement of the disk disorders; GIIl: arthralgia, arthiris and arthosis diagnosis; GEE Model: binomial distribution with logit link, robust estimator at covariance matrix and exchangeable correlation matrix; ${ }^{*}$ different lowercase letter on lines (intra group analisys) denote significant difference $(p<0.05)$.

and arthrogenic TMD (Table 2). At D90 (follow-up) a modification in the TMD Axis I classification of patients treated with PBM and MT was observed.

\section{Axis I: assessment of jaw movements}

Table 3 displays the data on jaw movements at D0, D28, and D90 according to the treatment group. In the mouth opening evaluation, GPBM showed significant enhancement between D0 and D28 ( $p<0.01)$. GMT presented an improvement comparing D0 to D28 $(\mathrm{p}<0.01)$ and D0 to D90 ( $\mathrm{p}=0.02)$. Maximum opening analysis revealed that GMT and GCT promoted improvements between D0 and D90 ( $p=0.023$ and $p=0.05$, respectively). Right excursion in GPBM showed improvement in the movement between D0 and D28.

No difference was observed in maximum opening with assistance, protrusion, and left excursion movements during the time and among the treatments. 
- Comparative effectiveness of photobiomodulation and manual therapy alone or combined in TMD patients: a randomized clinical

Table 3. RDC Axis I: Assessment of jaws movements $(\mathrm{mm})$ at baseline, end of the treatment, and follow up of patients treated with different protocols. Média (IC95\%)

\begin{tabular}{|c|c|c|c|c|}
\hline Group & Baseline & End Treatment & Follow up & p Time \\
\hline \multicolumn{5}{|c|}{ Opening' } \\
\hline PBM & $29.64^{\circ}[27.17-32.35]$ & 32.57 ^[29.53-35.92] & $36.86^{\mathrm{ab}}[34.51-39.36]$ & $<0.001$ \\
\hline MT & 27.92 ac[24.97-31.23] & $34.00^{b}[30.85-37.47]$ & 33.38 c[30.78-36.21] & \\
\hline CT & $29.71^{\circ a}[25.49-34.64]$ & $31.50^{\circ}[29.27-33.90]$ & 31.93 ॰[30.41-33.52] & \\
\hline \multicolumn{5}{|c|}{ Maximum opening 2} \\
\hline PBM & 40.86 ×[37.62-44.09] & $42.79 \square[40.15-45.42]$ & $44.50 \circ[42.24-46.76]$ & $<0.001$ \\
\hline MT & 38.46 व[35.06-41.86] & 41.85 [ [39.60-44.09] & 42.54 b[39.56-45.51] & \\
\hline CT & $35.29^{a}[31.03-39.54]$ & $38.93^{\circ}[36.70-41.16]$ & $39.93^{b}[37.86-42.00]$ & \\
\hline \multicolumn{5}{|c|}{ Maximun opening with assistance ${ }^{2}$} \\
\hline PBM & $46.79[43.61-49.96]$ & $45.64[43.40-47.89]$ & 46.14 [43.53-48.75] & 0.32 \\
\hline MT & 43.31 [39.68-46.93] & $46.31[43.77-48.84]$ & 44.62 [41.73-47.50] & \\
\hline СТ & 40.14 [35.51-44.78] & $42.79[40.21-45.36]$ & 42.07 [39.63-44.51] & \\
\hline \multicolumn{5}{|c|}{ Protrusion ${ }^{2}$} \\
\hline PBM & $9.57[7.43-11.71]$ & 8.79 [7.25-10.32] & 8.36 [7.08-9.63] & 0.28 \\
\hline MT & 8.08 [6.84-9.31] & 7.54 [5.37-9.71] & $7.15[5.10-9.21]$ & \\
\hline СТ & 7.71 [5.65-9.78] & 9.36 [8.26-10.45] & 7.79 [6.34-9.23] & \\
\hline \multicolumn{5}{|c|}{ Right excursion' } \\
\hline PBM & $5.93^{\circ}[4.26-8.26]$ & $8.36^{b}[7.21-9.68]$ & $7.90 \mathrm{ab}[6.69-9.33]$ & 0.03 \\
\hline MT & 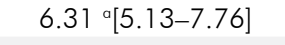 & 8.62 a[6.91-10.74] & $8.31^{a}[7.06-9.78]$ & \\
\hline CT & 6.57 a[5.21-8.29] & 6.64 a[5.63-7.83] & 7.36 a[6.57-8.24] & \\
\hline \multicolumn{5}{|c|}{ Left excursion' } \\
\hline PBM & 6.86 [4.71-9.99] & 8.00 [6.70-9.55] & 8.39 [7.05-9.99] & 0.281 \\
\hline MT & 7.00 [5.09-9.63] & $8.77[7.04-10.92]$ & 8.15 [7.35-9.05] & \\
\hline CT & 7.64 [6.07-9.63] & 7.71 [6.87-8.66] & 7.71 [6.85-8.69] & \\
\hline
\end{tabular}

GEE Model ( $p=, 0.05)$; 1 - Normal distribution with identity; 2- Gamma distribution with log link

*Different lowercase letters on lines (intra group analisys) denote significant difference $(p<0.05)$.

Table 4. RDC axis II: Assessment of D1 (intensity of chronic pain and disability).

\begin{tabular}{|c|c|c|c|c|c|}
\hline \multirow{2}{*}{ Variable } & \multicolumn{2}{|c|}{ Baseline } & \multicolumn{2}{|c|}{ Follow up } & \multirow{2}{*}{$\mathrm{p}$-value } \\
\hline & Low Incapacity & Higher Incapacity & Low Incapacity & Higher Incapacity & \\
\hline $\operatorname{PBM}(n=14)$ & $10(71 \%)$ & $4(29 \%)$ & $13(92.9 \%)$ & $1(7.1 \%)$ & 0.05 \\
\hline$M T(n=13)$ & $12(92.3 \%)$ & $1(7.7 \%)$ & 13 (100\%) & $0(0.0 \%)$ & 0.30 \\
\hline$C T(n=14)$ & 7 (50\%) & 7 (50\%) & $8(57.1 \%)$ & $6(42.9 \%)$ & 0.56 \\
\hline
\end{tabular}

GEE Model: binomial distribution with logit link and exchangeable correlation.

Table 5. RDC axis II: Assessment of D2 (degree of depression).

\begin{tabular}{lccccc}
\hline \multirow{2}{*}{ Varible } & \multicolumn{3}{c}{ Baseline } & \multicolumn{2}{c}{ Follow up } \\
\cline { 2 - 4 } & No depression & Moderate/Severe depression & No depression & Moderate/Severe depression & $p$-value \\
\hline PBM $(n=14)$ & $6(42.9 \%)$ & $8(57.1 \%)$ & $11(78.6 \%)$ & $3(21.4 \%)$ & 0.01 \\
MT $(n=13)$ & $7(53.8 \%)$ & $6(46.2 \%)$ & $9(69.2 \%)$ & $4(30.8 \%)$ & 0.30 \\
CT $(n=14)$ & $3(21.4 \%)$ & $11(78.6 \%)$ & $9(64.3 \%)$ & $5(35.7 \%)$ & $<0.001$ \\
\hline
\end{tabular}

GEE Model: binomial distribution with logit link and exchangeable correlation matrix.

Table 6. RDC axis II: Assessment of D3a (nonspecific physical symptoms with pain).

\begin{tabular}{lccccc}
\hline \multirow{2}{*}{ Variable } & \multicolumn{3}{c}{ Baseline } & \multicolumn{2}{c}{ Follow up } \\
\cline { 2 - 5 } & Normal & Moderate/Severe symptoms & Normal & Moderate/Severe symptoms & p-value \\
\hline PBM $(n=14)$ & $3(21.4 \%)$ & $11(78.6 \%)$ & $7(50 \%)$ & $7(50 \%)$ & 0.02 \\
MT $(n=13)$ & $5(38.5 \%)$ & $8(61.5 \%)$ & $10(23.3 \%)$ & $3(23.1 \%)$ & $<0.01$ \\
CT $(n=14)$ & $2(14.2 \%)$ & $12(85.7 \%)$ & $6(42.9 \%)$ & $8(57.1 \%)$ & 0.02 \\
\hline
\end{tabular}

GEE Model: binomial distribution with logit link and exchangeable correlation matrix. 
Table 7. RDC axis II: Assessment of D3b nonspecific physical symptoms without pain).

\begin{tabular}{lccccc}
\hline \multirow{2}{*}{ Variable } & \multicolumn{3}{c}{ Baseline } & \multicolumn{2}{c}{ Follow up } \\
\cline { 2 - 4 } & Normal & Moderate/Severe symptoms & Normal & Moderate/Severe symptoms & p-value \\
\hline PBM $(n=14)$ & $5(35.7 \%)$ & $9(64.2 \%)$ & $8(57.1 \%)$ & $6(42.9 \%)$ & 0.05 \\
MT $(n=13)$ & $6(46.2 \%)$ & $7(53.8 \%)$ & $11(84.6 \%)$ & $2(15.3 \%)$ & $<0.01$ \\
CT $(n=14)$ & $2(14.2 \%)$ & $12(85.7 \%)$ & $8(57.1 \%)$ & $6(42.9 \%)$ & $<0.01$ \\
\hline
\end{tabular}

GEE Model: binomial distribution with logit link and exchangeable correlation matrix.

Table 8. RDC axis II: Analysis of jaw disability (D4) at baseline and follow up.

\begin{tabular}{|c|c|c|c|}
\hline \multirow{2}{*}{ Group } & Baseline & Follow & \multirow{2}{*}{$\mathrm{p}$-value } \\
\hline & média (IC95\%) & média (IC95\%) & \\
\hline \multicolumn{4}{|c|}{ Chewing } \\
\hline PBM & $0.50 a[0.26-0.74]$ & $0.29 a[0.11-0.56]$ & $<0.001$ \\
\hline MT & $0.85 a[0.55-0.96]$ & $0.38 b[0.17-0.66]$ & \\
\hline CT & $0.93 a[0.63-0.99]$ & $0.57 a[0.32-0.79]$ & \\
\hline \multicolumn{4}{|c|}{ Exercising } \\
\hline PBM & $0.07 a[0.01-0.37]$ & $0.00 a[0.00-0.00]$ & $<0.001$ \\
\hline MT & $0.08 a[0.01-0.39]$ & $0.08 a[0.01-0.39]$ & \\
\hline $\mathrm{CT}$ & $0.50 a[0.26-0.74]$ & $0.07 b[0.01-0.37]$ & \\
\hline \multicolumn{4}{|c|}{ Eating hard foods } \\
\hline PBM & $0.71 a[0.44-0.89]$ & $0.43 b[0.21-0.68]$ & 0.001 \\
\hline MT & $0.85 a[0.55-0.96]$ & $0.54 b[0.28-0.78]$ & \\
\hline $\mathrm{CT}$ & $0.93 a[0.63-0.99]$ & $0.71 a[0.44-0.89]$ & \\
\hline \multicolumn{4}{|c|}{ Eating soft foods } \\
\hline PBM & $0.07 a[0.01-0.37]$ & $0.00 a[0.00-0.00]$ & $<0.001$ \\
\hline MT & $0.23 a[0.08-0.52]$ & $0.00 b[0.00-0.00]$ & \\
\hline CT & $0.36 a[0.16-0.62]$ & $0.14 a[0.04-0.43]$ & \\
\hline \multicolumn{4}{|c|}{ Smiling/laughing } \\
\hline PBM & $0.36 a[0.16-0.62]$ & $0.07 b[0.01-0.37]$ & 0.004 \\
\hline MT & $0.31 a[0.12-0.59]$ & $0.08 b[0.01-0.39]$ & \\
\hline CT & $0.50 a[0.26-0.74]$ & $0.36 a[0.16-0.62]$ & \\
\hline \multicolumn{4}{|c|}{ Swallowing } \\
\hline PBM & $0.14 a[0.04-0.43]$ & $0.07 a[0.01-0.37]$ & $<0.001$ \\
\hline MT & $0.23 a[0.08-0.52]$ & $0.00 b[0.00-0.00]$ & \\
\hline $\mathrm{CT}$ & $0.43 a[0.21-0.68]$ & $0.21 a[0.07-0.49]$ & \\
\hline
\end{tabular}

GEE Model: binomial distribution with logit link and exchangeable correlation matrix.

*Different lowercase letters on lines (intra group analisys) denote significant difference $(p<0.05)$

therapeutic possibilities have been described for symptom relief and jaw movement improvements. 5,6,10,12 Occlusal splints are usually the first choice among dentists, but in our study we evaluated two therapies of low cost and easy application, the PBM and MT, isolated or combined. Our main results showed that PBM, MT, and CT were effective in reducing pain, improving the range of some mandibular movements and masticatory functions, enhancing non-specific physical symptoms, and reducing depression and
Table 9. Mean Beck anxiety inventory (BAI) scores in all groups at baseline and follow up.

\begin{tabular}{lccc}
\hline \multirow{2}{*}{ Variable } & Baseiline & Follow up & \\
\cline { 2 - 3 } & Mean (IC95\%) & Mean (IC95\%) & \\
\hline PBM & $17,86^{a}[8.56-27.15]$ & $9.57^{b}[2.01-17.14]$ & 0.02 \\
MT & $14.92^{a}[6.31-23.54]$ & $7.92^{b}[3.93-11.91]$ & 0.03 \\
CT & $31.21^{a}[21.93-40.49]$ & $13.57^{b}[8.54-18.60]$ & $<0.001$ \\
\hline
\end{tabular}

GEE Model: normal distribution with identity link; *Different lowercase letters on lines (intra group analisys) denote significant difference $(p<0.05)$.

anxiety symptoms. However, a comparative analysis of all variables among groups did not show significant differences. Some of these results were expected based on the fact that PBM and MT are noninvasive methods previously used in other studies with successful pain control, ${ }^{7,17,18}$ and mandibular movement enhancement. ${ }^{1,6,7,12}$ However, they have not been analyzed in myogenic and arthrogenic TMD patients as a combined therapy. Moreover, our results were the first to demonstrate that both therapies can improve other important aspects related to TMD such as psychological and psychosocial factors.

In this clinical trial, RDC/TMD Axis I and II, VAS, and BAI were used for a complete assessment of TMD at baseline and post-treatment periods, all of which are internationally validated tools. Specifically, RDC/TMD is an important instrument for TMD diagnosis, presenting good validity and reliability. ${ }^{2}$ Using the RDC/TMD Axis I, myogenic and arthrogenic TMD were diagnosed in all 51 patients included. Also, the main characteristics of our sample are in accordance with the literature, which shows a predominance of females exhibiting pain, jaw function limitation, some degree of chronic pain, and depression and anxiety symptoms., ${ }^{4,78,19,20}$ These are important aspects that were neglected by most studies evaluating TMD treatment by VAS 
and mouth opening analysis. ${ }^{6,20,21}$ However, it is well known that TMD patients often present significant psychological distress, so we decided to use RDC/ TMD Axis II and BAI questionnaire to better analyze the effect of therapies used in this study regarding psychosocial and anxiety factors. ${ }^{2,4,7}$

PBM has been widely used to treat several inflammatory conditions. ${ }^{7,11}$ The most common effect is the improvement of local blood circulation, modulation of inflammation, pain control, and tissue repair. ${ }^{1718,22}$ In the present study, we observed several positive effects of PBM alone and combined with MT. However, the clinical efficacy of PBM in TMDs has been considered controversial, probably due to the differences in parameters and dosages used, application sites, and evaluation and classification criteria for this condition. In agreement with our results, some authors report PBM efficacy in increasing range of motion of the jaw and reducing pain, 1,21,23 compared to placebo and other therapies. Differently, other authors describe no significant difference between PBM and placebo. ${ }^{8,24,25}$ Also according to our results, and similarly to other previous studies, PBM can be beneficial to the patient by helping in pain relief, promotion of comfort, and improving jaw movements and psychological aspects. Additionally, we observed that the positive effects of PBM were maintained at D60, in accordance to other studies. ${ }^{20,24}$ Our study was the first to demonstrate that PBM is similar to MT in the treatment of TMD. Other modalities were compared with PBM by other authors and showed similar results to transcutaneous electrical neural stimulation (TENS) ${ }^{6}$ and occlusal splint ${ }^{17}$.

This clinical trial also used MT, which has been considered an effective option for TMD treatment. ${ }^{10,26}$ Our results revealed that MT promotes pain relief, improvement of jaw movements as well as a decrease of anxiety symptoms. These results could be justified by the fact that tissue manipulation increases fibrocartilage nutrition and muscle relaxation and range of movement. ${ }^{5,27}$ In addition, muscles and ligaments involving the TMJ subjected to massage, traction, and mobilization, tend to be relax, improving vascularization and tissue compliance, decreasing pain, and improving movement range. ${ }^{26}$ In a literature review, Santos and Pereira ${ }^{28}$ evaluated the effectiveness of manual techniques in the treatment of patients with TMD, and the results were positive when the procedures were applied alone or associated with other therapeutic resources, corroborating the importance of MT in TMD treatment.

Based on the multifactorial etiology of TMD, it has been suggested that the association of different techniques can present better results in treating TMDs, by maximizing any complementary effects. ${ }^{6,10,12,13,26}$ At the beginning of this study we considered the hypothesis that CT would present superior results to those of the isolated therapies. However, since all therapies presented significant results in the evaluated subjects, our hypothesis was not supported. One possible explanation for this is that both PBM and MT act by promoting vasodilatation, which can account for positive effects in muscle spasms and pain, thus recovering normal function.

The association of psychosocial aspects with the severity and persistence of clinical symptoms of TMD can influence the patient's response to treatments, hindering the performance of daily activities. ${ }^{2}$ Despite this, few studies report the impact of treatments on the improvement of these aspects, which could be evaluated in detail using RDC/TMD Axis II and a BAI questionnaire. ${ }^{7}$ RDC/TMD Axis II enables the identification of emotional conditions and the impact of TMD on the daily life of affected individuals, but the questionnaire does not allow a clinical psychiatric diagnosis of such individuals. In the present clinical trial, we observed that none of the treatments induced modification in the intensity of chronic pain (D1). This result is probably due to the RDC/TMD (Axis II) questions used to classify the degree of chronic pain are the means of pain intensity reported in 6 months. Since the duration of this study was less than 6 months, little difference can be noticed in relation to this classification.

Depression (D2) symptoms decreased after PBM and CT treatments. At baseline, $78.6 \%$ of CT patients had moderate to severe degrees of depression compared to $57.1 \%$ of patients in PBM. At follow-up, only $35.7 \%$ of the CT group and $21.4 \%$ of the PBM group maintained this classification. MT presented a non-significant reduction in severity of depression (from $46 \%$ to $30.8 \%$ ) but this group presented a lower 
number of patients with moderate/severe depression at baseline. In addition, we observed that all treatments promoted reduction in physical symptoms with pain (D3a). This result is in agreement with the VAS analysis and with Rodrigues et al., ${ }^{7}$ study who also observed that PBM promotes an improvement in this function. For physical symptoms without pain (D3b), there was a significant improvement in all groups, again in accordance with the results of Rodrigues et al.. ${ }^{7}$

Another important aspect evaluated by RDC/ TMD Axis II is the functional deficit of TMD patients in daily activities (D4). Here, we demonstrated a significant improvement in 5 functions with MT (chewing, hard and soft food intake, smiling, and swallowing), 2 functions with PBM (ingestion of hard foods and smiling), and 1 with CT (exercise performance). The positive effects of PBM in these features was previously reported by Rodrigues et al. ${ }^{7}$ Unfortunately, MT alone or combined with other therapies was never evaluated before by RDC/TMD Axis II, which makes it difficult to compare our results. In general, our results demonstrated that depression, physical symptoms, and jaw functions need to be evaluated because they can indicate other important effects of TMD treatment in patients.

Anxiety symptoms are another aspect that directly influence physical symptoms of TMD. Currently, BAI is considered a standard for assessing anxiety by rating it at minimum, mild, moderate, and severe levels. ${ }^{2}$ Here, we observed that at baseline patients presented moderate and severe levels and after therapies an improvement was detected. It is widely accepted that TMD symptoms fluctuate and are self-limiting, ${ }^{11}$ which means that many patients will exhibit a natural and expected improvement, even if no therapy is offered. However, in the present study, we used different tools to analyze TMD patients, trying to cover the most important clinical and physiological aspects involved with the development and maintenance of this disease. Analyzing the totality of our data, we can verify that all the therapies used promoted improvement in several signs and symptoms of TMD. The most relevant limitation of this clinical trial is the absence of a placebo group. However, it is not possible to perform a MT placebo group; in addition, a placebo for patients with chronic diseases such as TMD would not be approved by the ethics committee.

\section{Conclusion}

In conclusion, the present findings indicated that all protocols tested were able to decrease pain and improve mandibular movements and some mandibular functions, reducing negative effects of the psychosocial aspects and the anxiety symptoms of TMD patients. Moreover, results showed that therapies improve functional capacity and the resume of daily activities. However, a combination of PBM and MT did not promote an increase in the effect of both therapies alone.

\section{References}

1. Ahrari F, Madani AS, Ghafouri ZS, Tunér J. The efficacy of low-level laser therapy for the treatment of myogenous temporomandibular joint disorder. Lasers Med Sci. 2014 Mar;29(2):551-7. https://doi.org/10.1007/s10103-012-1253-6

2. Suvinen TI, Reade PC, Kemppainen P, Könönen M, Dworkin SF. Review of aetiological concepts of temporomandibular pain disorders: towards a biopsychosocial model for integration of physical disorder factors with psychological and psychosocial illness impact factors. Eur J Pain. 2005 Dec;9(6):613-33. https://doi.org/10.1016/i.ejpain.2005.01.012

3. Manfredini D, Guarda-Nardini L, Winocur E, Piccotti F, Ahlberg J, Lobbezoo F. Research diagnostic criteria for temporomandibular disorders: a systematic review of

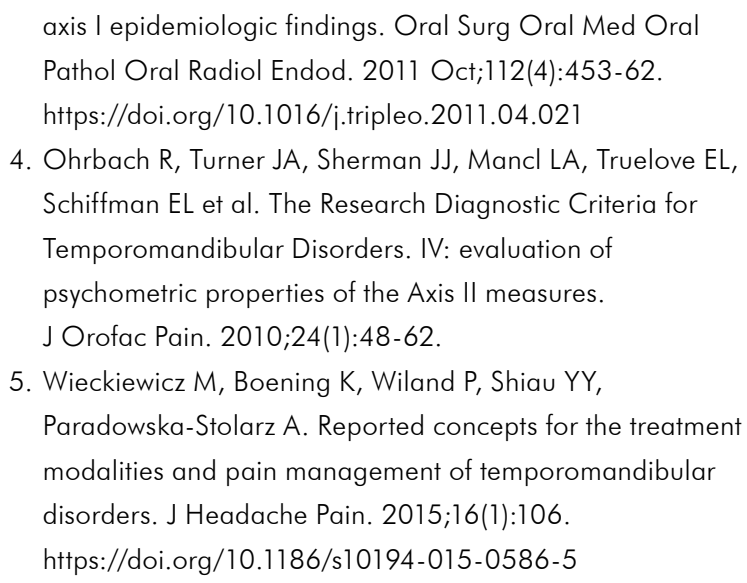


6. Kato MT, Kogawa EM, Santos CN, Conti PC. TENS and lowlevel laser therapy in the management of temporomandibular disorders. J Appl Oral Sci. 2006 Apr;14(2):130-5. https://doi.org/10.1590/S1678-77572006000200012

7. Rodrigues JH, Marques MM, Biasotto-Gonzalez DA, Moreira MS, Bussadori SK, Mesquita-Ferrari RA et al. Evaluation of pain, jaw movements, and psychosocial factors in elderly individuals with temporomandibular disorder under laser phototherapy. Lasers Med Sci. 2015 Apr;30(3):953-9. https://doi.org/10.1007/s10103-013-1514-z

8. Cunha LA, Firoozmand LM, Silva AP, Esteves SA, Oliveira W. Efficacy of low-level laser therapy in the treatment of temporomandibular disorder. Int Dent J. 2008 Aug;58(4):2137. https://doi.org/10.1111/i.1875-595X.2008.tb00351.x

9. Carrasco TG, Mazzetto MO, Mazzetto RG, Mestriner W Jr. Low intensity laser therapy in temporomandibular disorder: a phase II double-blind study. Cranio. 2008 Oct;26(4):274-81. https://doi.org/10.1179/crn.2008.037

10. Furto ES, Cleland JA, Whitman JM, Olson KA. Manual physical therapy interventions and exercise for patients with temporomandibular disorders. Cranio. 2006 Aug;24(4):283-91. https://doi.org/10.1179/crn.2006.044

11. Hamblin MR. Mechanisms and mitochondrial redox signaling in photobiomodulation. Photochem Photobiol. 2018 Mar;94(2):199-212. https://doi.org/10.1111/php.12864

12. Ismail F, Demling A, Hessling K, Fink M, StieschScholz M. Short-term efficacy of physical therapy compared to splint therapy in treatment of arthrogenous TMD. J Oral Rehabil. 2007 Nov;34(11):807-13. https://doi.org/10.1111/j.1365-2842.2007.01748.x

13. Núñez SC, Garcez AS, Suzuki SS, Ribeiro MS. Management of mouth opening in patients with temporomandibular disorders through low-level laser therapy and transcutaneous electrical neural stimulation. Photomed Laser Surg. 2006 Feb;24(1):45-9. https://doi.org/10.1089/pho.2006.24.45

14. Biasotto-Gonzalez DA. Bérzin F. Electromyographic study of patients with masticatory muscles disorders, physiotherapeutic (massage). Braz J Oral Sci. 2004 Jan;3(10):516-21.

15. Cetiner S, Kahraman SA, Yücetaş S. Evaluation of low-level laser therapy in the treatment of temporomandibular disorders. Photomed Laser Surg. 2006 Oct;24(5):637-41. https://doi.org/10.1089/pho.2006.24.637

16. Shirani AM, Gutknecht N, Taghizadeh M, Mir M. Low-level laser therapy and myofacial pain dysfunction syndrome: a randomized controlled clinical trial. Lasers Med Sci. 2009 Sep;24(5):715-20. https://doi.org/10.1007/s10103-008-0624-5

17. Ferreira LA, Oliveira RG, Guimarães JP, Carvalho AC, De Paula MV. Laser acupuncture in patients with temporomandibular dysfunction: a randomized controlled trial. Lasers Med Sci. 2013 Nov;28(6):1549-58. https://doi.org/10.1007/s10103-013-1273-x

18. Mazzetto MO, Hotta TH, Pizzo RC. Measurements of jaw movements and TMJ pain intensity in patients treated with GaAlAs laser. Braz Dent J. 2010;21(4):356-60. https://doi.org/10.1590/S0103-64402010000400012

19. Carvalho CM, Lacerda JA, Santos Neto FP, Cangussu MC, Marques AM, Pinheiro AL. Wavelength effect in temporomandibular joint pain: a clinical experience. Lasers Med Sci. 2010 Mar;25(2):229-32. https://doi.org/10.1007/s10103-009-0695-y

20. Sayed N, Murugavel C, Gnanam A. Management of temporomandibular disorders with low level laser therapy. J Maxillofac Oral Surg. 2014 Dec;13(4):444-50. https://doi.org/10.1007/s12663-013-0544-1

21. Dostalová T, Hlinakova P, Kasparova M, Rehacek A, Vavrickova L, Navrátil L. Effectiveness of physiotherapy and GaAlAs laser in the management of temporomandibular joint disorders. Photomed Laser Surg. 2012 May;30(5):275-80. https://doi.org/10.1089/pho.2011.3171

22. Emshoff R, Bösch R, Pümpel E, Schöning H, Strobl H. Low-level laser therapy for treatment of temporomandibular joint pain: a double-blind and placebo-controlled trial. Oral Surg Oral Med Oral Pathol Oral Radiol Endod. 2008 Apr;105(4):452-6. https://doi.org/10.1016/j.tripleo.2007.09.012

23. Sancakli E, Gökçen-Röhlıg B, Balık A, Öngül D, Kıpırdı $\mathrm{S}$, Keskın H. Early results of low-level laser application for masticatory muscle pain: a double-blind randomized clinical study. BMC Oral Health. 2015 Oct;15(1):131. https://doi.org/10.1186/s12903-015-0116-5

24. Panhoca VH, Lizarelli RF, Nunez SC, Pizzo RC, Grecco C, Paolillo FR et al. Comparative clinical study of light analgesic effect on temporomandibular disorder (TMD) using red and infrared led therapy. Lasers Med Sci. 2015 Feb;30(2):815-22. https://doi.org/10.1007/s10103-013-1444-9

25. Salmos-Brito JA, Menezes RF, Teixeira CE, Gonzaga RK, Rodrigues BH, Braz R et al. Evaluation of low-level laser therapy in patients with acute and chronic temporomandibular disorders. Lasers Med Sci. 2013 Jan;28(1):57-64. https://doi.org/10.1007/s10103-012-1065-8

26. Gomes CA, El Hage Y, Amaral AP, Politti F, BiasottoGonzalez DA. Effects of massage therapy and occlusal splint therapy on electromyographic activity and the intensity of signs and symptoms in individuals with temporomandibular disorder and sleep bruxism: a randomized clinical trial. Chiropr Man Therap. 2014 Dec;22(1):43. https://doi.org/10.1186/s12998-014-0043-6

27. Kalamir A, Pollard H, Vitiello A, Bonello R. Intra-oral myofascial therapy for chronic myogenous temporomandibular disorders: a randomized, controlled pilot study. J Manual Manip Ther. 2010 Sep;18(3):139-46. https://doi.org/10.1179/106698110X12640740712374

28. Santos LFS, Pereira MCA The effectiveness of manual therapy in the treatment of temporomandibular disorders (TMD): a review of the literature. Rev Aten Saúde. 2016 Jan;14(49):72-7. https://doi.org/10.13037/rbcs.vol14n49.3596 